\title{
BEST UNIFORM APPROXIMATIONS VIA ANNIHILATING MEASURES
}

\author{
BY WILLIAM HINTZMAN
}

Communicated by R. A. Kunze, March 5, 1970

The problem under consideration in this paper is that of uniformly approximating an arbitrary continuous function $g$ on the closed unit disk $\bar{D}$ by continuous functions $f$ which are analytic in $D=\{z$ complex: $|z|<1\}$. In particular, we are concerned with the existence, uniqueness, and construction of a best approximation $f_{0}$ to $g$. Our results consist of a proof of the uniqueness of $f_{0}$ when it exists and an algorithm for constructing $f_{0}$ for certain classes of functions $g$. Both results follow from a more general theorem on best uniform approximations and annihilating measures.

If $E$ is a normed linear space, $A$ is a subspace of $E$, and $S_{A}^{*}$ consists of all the linear functionals $L$ on $E$ with $\|L\| \leqq 1$ and which vanish on $A$ then, as a consequence of the Hahn-Banach theorem, the following relationship holds [1].

Theorem 1. If $g \in E$ then

$$
\|g\|_{A}=\inf _{\delta \in A}\|g-f\|=\max _{L \in S_{\boldsymbol{A}}^{*}}|L(g)| .
$$

For $E=C(K)$, the continuous complex valued functions defined on the compact Hausdorff space $K$, additional information can be obtained from Theorem 1 by applying the Riesz representation theorem [4] to $L \in S_{A}^{*}$. Here $\|g\|=\max _{z} \in K|g(z)|$ is the uniform norm.

THEOREM 2. If $g \in C(K), f_{0} \in A$ is a best uniform approximation to $g, L \in S_{A}^{*}$, and $L(g)=\|g\|_{A}$ then $g-f_{0}=\|g\|_{A} \phi$ a.e. $d \mu$ where $\phi d \mu$ is the polar decomposition of the unique regular Borel measure on $K$ which represents $L$.

Proof. By Theorem 1, there is an $L \in S_{A}^{*}$ with $L(g)=\|g\|_{A}$ and $\|L\|=1$. Let $\phi d \mu$ be the measure which represents $L$ where $|\phi|=1$ a.e. $d \mu, d \mu \geqq 0$ and $\int_{K} d \mu=1$. Now,

$$
\|g\|_{A}=\int_{K}\left(g-f_{0}\right) \phi d \mu \leqq \int_{K}\left|\left(g-f_{0}\right) \phi\right| d \mu \leqq \int_{K}\left\|g-f_{0}\right\| d \mu=\|g\|_{A} .
$$

AMS 1969 subject classifications, Primary 4130, 4140; Secondary 4215, 4625.

Key words and phrases. Best approximation, uniform norm, analytic functions, harmonic functions, linear functional, annihilating measure, extreme point. 
Therefore,

$$
\int_{K}\left(g-f_{0}\right) \phi d \mu=\int_{K}\left|\left(g-f_{0}\right) \phi\right| d \mu=\|g\|_{A} .
$$

Since $\left|\left(g-f_{0}\right)\right| \leqq\|g\|_{A}$ we must have $\left|g-f_{0}\right|=\|g\|_{A}$ on the support of $d \mu$.

Then it follows that

$$
\left(g-f_{0}\right) \phi=\|g\|_{A} \text { a.e. } d \mu
$$

and

$$
g-f_{0}=\|g\|_{A} \Phi \text { a.e. } d \mu
$$

which was to be proved.

Let $K=\bar{D}$, the closed unit disk, and let $A$ consist of all functions in $C(\bar{D})$ which are analytic in $D$. Then the support of the measure $d \mu$ in Theorem 2 is large enough to ensure the uniqueness of $f_{0}$ when $f_{0}$ exists.

THEOREM 3. If $f_{0} \in A$ is a best uniform approximation to $g \in C(\bar{D})$ then $f_{0}$ is unique.

Proof. Suppose $f_{0}$ is not unique. Then there is an $f_{1} \in A, f_{1} \neq f_{0}$ such that $\left\|g-f_{0}\right\|=\left\|g-f_{1}\right\|=\|g\|_{A}$. Let $\phi d \mu \in S_{A}^{*}$ be the measure in Theorem 2. Then $f_{0}=f_{1}=g-\|g\|_{A} \bar{\phi}$ a.e. $d \mu$ and $h=f_{1}-f_{0}=0$ on $K$, the support of $\mu$. Therefore $K \cap D$ can not have a limit point in $D$ so $K \cap D$ is at most countable.

One can now show that $A$ is dense in $L^{2}(d \mu, K \cap D)$ and therefore $\phi d \mu$ is the zero measure on $K \cap D$. Then, by the F. and M. Riesz theorem [3], $\phi d \mu$ is absolutely continuous with respect to Lebesgue measure on $\partial D$. But $f_{0}=f_{1}$ on $\bar{D}$ if $K \cap \partial D$ has positive Lebesgue measure [3]. Therefore, $K \cap \partial D$ has zero Lebesgue measure and $g \in A$ so that $g=f_{0}=f_{1}$ which contradicts our assumption.

We now demonstrate the existence of best approximations $f_{0}$ in $A$ to harmonic functions of the form $g=\sum_{j=0}^{n} b_{j} \bar{z}^{j+1}$. The maximum modulus principle for harmonic functions implies that we may restrict our attention to approximating $g$ on $\partial D$ by $f$ in $A$. The linear functionals that annihilate $A$ on $\partial D$ are of the form

$$
L(h)=\int_{0}^{2 \pi} h\left(e^{i \theta}\right) e^{i \theta} f\left(e^{i \theta}\right) \frac{d \theta}{2 \pi}
$$

where $f \in H^{1}$, the Hardy space $H^{1}$, i.e., $f$ is an $L^{1}$ function on $\partial D$ whose harmonic extension to $D$ is analytic. We will simply write $L(h)$ 
$=\int z h f(d \theta / 2 \pi)$ for the above integral. Theorem 1 then says

$$
\|g\|_{A}=\max _{f \in H^{1}} \frac{\left|\int z g f(d \theta / 2 \pi)\right|}{\|f\|_{1}} .
$$

However, $\|g\|_{A}$ may be calculated by considering a much smaller class of linear functionals.

LEMmA. If $g=\sum_{j=0}^{n} b_{j} z^{j+1}$ then

$$
\|g\|_{A}=\max _{f \text { outer; } f \in P_{n}} \frac{\left|\left(f^{2}, \bar{z} \bar{g}\right)\right|}{(f, f)}
$$

where $\left(f^{2}, \bar{z} \bar{g}\right)$ and $(f, f)$ are inner products in $L^{2}(d \theta / 2 \pi, \partial D)$.

Proof. Rudin and de Leeuw have shown that if $f \in H^{1}$ and $\|f\|_{1}=1$ then $f=\frac{1}{2}\left(f_{1}+f_{2}\right)$ where $f_{1}$ and $f_{2}$ are outer functions in $H^{1}$ with $L_{1^{-}}$ norms $=1[2]$. Hence (1) reduces to

$$
\|g\|_{A}=\max _{f \text { outer } ; f \in H^{1}} \frac{|(f, \bar{z} \bar{g})|}{\|f\|_{1}} .
$$

But $f$ being outer in $H^{1}$ implies that $f^{1 / 2}$ is outer in $H^{2}$. Therefore,

$$
\|g\|_{A}=\max _{f \text { outer } ; f \in H^{2}} \frac{\left|\left(f^{2}, \bar{z} \bar{g}\right)\right|}{(f, f)} .
$$

Let $P_{n}$ denote the space of all polynomials in $z$ of degree $\leqq n$ and $f_{n}$ denote the $L^{2}(d \theta / 2 \pi)$ projection of $f$ onto $P_{n}$. Then

$$
\frac{\left|\left(f^{2}, \bar{z} \bar{g}\right)\right|}{(f, f)} \leqq \frac{\left|\left(f_{n}^{2}, \bar{z} \bar{g}\right)\right|}{\left(f_{n}, f_{n}\right)}
$$

since the numerators are equal and $(f, f) \geqq\left(f_{n}, f_{n}\right)$. Since the inequality is strict for $f \notin P_{n}$ we have

$$
\|g\|_{A}=\max _{f \text { outer; } f \in P_{n}} \frac{\left|\left(f^{2}, \bar{z} \bar{g}\right)\right|}{(f, f)} .
$$

THEOREM 4. If $g=\sum_{j=0}^{n} b_{j} \bar{z}^{j+1}$ then there is a rational function $f_{0}$ in $A$ which is the unique best approximation to $\mathrm{g}$.

Proof. Applying the lemma, there is an $f$ in $P_{n}, f$ outer, with $(f, f)$ $=1$ and $\|g\|_{A}=\int z g f^{2}(d \theta / 2 \pi)$. The polar decomposition of the measure is $(z f / \bar{f})|f|^{2}(d \theta / 2 \pi)$ where $\phi=z f / \bar{f}$ and $d \mu=|f|^{2}(d \theta / 2 \pi)$. On $\partial D, \bar{\phi}$ $=\bar{z} \bar{f} / f$ has at most $n$ discontinuities which are removable so let $\bar{\phi}$ 
denote the modification of $\bar{z} \bar{f} / f$ which is continuous on $\partial D$. We claim that $f_{0}=g-\|g\|_{A} \bar{\phi}$ is the unique best approximation to $g$ from $A$.

To show that $f_{0} \in A$ consider a sequence $f_{n} \in A$ with $\left\|g-f_{n}\right\| \rightarrow\|g\|_{A}$. Then by either a normal family argument on $\left\{f_{n}\right\}$ or a weak* compactness argument [2] there is an $h \in H^{\infty}$ with $\|g-h\|_{\infty}=\|g\|_{A}$. Applying the proof of Theorem 2 we have $h=f_{0}$ a.e. $d \theta$ on $\partial D$. Consequently both functions have the same analytic extension to $D$ and hence $f_{0} \in A$.

Uniqueness of $f_{0}$ follows from Theorem 3 and the fact that $f_{0}$ is a rational function follows from our algorithm for calculating $f_{0}$ which we describe next.

Let $g, f$, and $f_{0}$ be as above where $f=\sum_{j=0}^{n} a_{j} z^{i}, \rho=\|g\|_{A},(f, f)=1$, and $\rho=\left(f^{2}, \bar{z} \bar{g}\right)$. Then $\left(g-f_{0}\right) z f / \bar{f}=\rho$ a.e. $d \theta$ or

(2) $z g f-z f_{0} f=\rho \bar{f}$ on $\partial D$.

The nonpositive Fourier coefficients in (2) satisfy the matrix equation

(3) $B F=\rho \bar{F}$ where

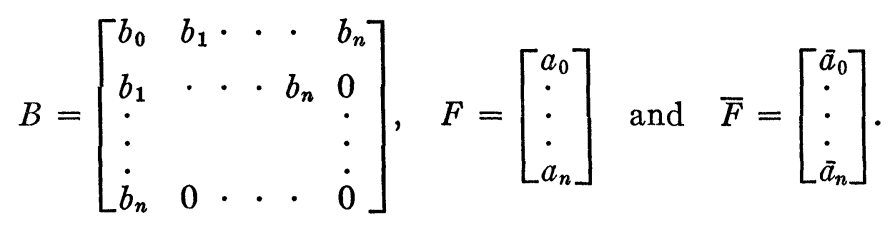

From (3) can be derived

(4) $\left(\bar{B} B-\rho^{2} I\right) \quad F=0$.

The method for finding $f_{0}$ consists of first finding the largest positive eigenvalue $\rho^{2}$ of $\bar{B} B$ and then solving $\left(\bar{B} B-\rho^{2} I\right) X=0$ for a nontrivial solution $X=F_{1}$. Then either $F_{1}$ or $(i)\left(\bar{B} \bar{F}_{1}-\rho F_{1}\right)$ is a nontrivial solution of $B \bar{X}=\rho X$. Let $F$ denote that nontrivial solution. Then $f$ is defined by $F$ and $f_{0}$ by $f_{0}=g-\rho \bar{z} \bar{f} / f$ on $\partial D$. Choosing $F$ so that $(F, F)=1$ shows that $\rho^{2}=\|g\|_{A}^{2}$ is the largest positive eigenvalue of $\bar{B} B$ since $F$ is a solution to both (3) and (4) and since $\int z g f^{2}(d \theta / 2 \pi)=F^{t} B F=\rho$, where $F^{t}$ is the transpose of $F$.

As an example of the preceding method consider $g=3 \bar{z}+2 \bar{z}^{2}$. Then

$$
B=\left[\begin{array}{ll}
3 & 2 \\
2 & 0
\end{array}\right] \text { and } \bar{B} B=\left[\begin{array}{rr}
13 & 6 \\
6 & 4
\end{array}\right] \text {. }
$$

$\operatorname{Det}\left(\bar{B} B-\rho^{2} I\right)=\left(\rho^{2}-16\right)\left(\rho^{2}-1\right)$. Hence $\|g\|_{A}=4$. Now $F=\left(\begin{array}{l}2 \\ 1\end{array}\right)$ is a solution of (3). Therefore, let $f=2+z$. Then

$$
f_{0}=g-4 \Phi=\frac{3 z+2}{z^{2}}-\frac{4}{z^{2}}\left(\frac{1+2 z}{z+2}\right) \text { on } \partial D .
$$


Thus $f_{0}=3 /(z+2)$ is the unique best approximation from $A$ to $g$ on $\bar{D}$.

\section{REFERENCES}

1. R. C. Buck, Applications of duality in approximation theory, Proc. Sympos. Approximation of Functions (General Motors Res. Lab., 1964) Elsevier, Amsterdam, 1965, pp. 27-42. MR 33 \#4554.

2. K. de Leeuw and W. Rudin, Extreme points and extremum problems in $H_{1}$, Pacific J. Math. 8 (1958), 467-485. MR 20 \#5426.

3. K. Hoffman, Banach spaces of analytic functions, Prentice-Hall Series in Modern Analysis, Prentice-Hall, Englewood Cliffs, N. J., 1962. MR 24 \#A2844.

4. W. Rudin, Real and complex analysis, McGraw-Hill, New York, 1966. MR 35 $\# 1420$.

San Diego State College, San Diego, California 92115 